\title{
Sirtuin 3: A Molecular Pathway Linking Sleep Deprivation to Neurological Diseases
}

\author{
Karim Fifel \\ Laboratory of Neurophysiology, Molecular Cell Biology Department, Leiden University Medical Center, 2300 RC Leiden, The Netherlands \\ Review of Zhang et al.
}

Insufficient sleep causes a multitude of devastating health problems and has severe societal consequences (Wade, 2010; Palma et al., 2013). Current estimates suggest that more than one-third of all adults receive less than $7 \mathrm{~h}$ of sleep per day (Perry et al., 2013). This troubling statistic is likely to remain unchanged-or may become even worse-in the near future, as many factors in our modern society prevent sufficient sleep. Given this situation, developing viable methods to deal with the negative consequences of sleep deprivation such as cognitive and metabolic disorders has become a top research priority.

Evidence has been mounting over the past 10 years suggesting a link between altered sleep patterns and several neurodegenerative diseases (Costandi, 2013). However, whether changes in sleep/wakefulness patterns are a cause or consequence of neurodegenerative processes has remained elusive. Moreover, the fact that sleep deprivation has widespread effects on many physiological systems (Palma et al., 2013) hampers the search for a well defined molecular pathway that links sleep loss with neurodegeneration.

Received May 7, 2014; revised May 31, 2014; accepted June 4, 2014.

K.F. received a post-doc fellowship from Fondation Fyssen. I thank Drs. J.H. Meijer and T. Deboer for their valuable inputs and improvements of the draft and Dr. C.F. Barrett for his English editing.

Correspondence should be addressed to Karim Fifel, Laboratory of Neurophysiology, Molecular Cell Biology Department, Leiden University Medical Center, P0 Box 9600 Mailbox S5-P. 2300 RC Leiden, The Netherlands. E-mail:K.Fifel@lumc.nl or fifel-k@hotmail.com.

DOI:10.1523/JNEUROSCI.1848-14.2014

Copyright $\odot 2014$ the authors $\quad 0270-6474 / 14 / 349179-03 \$ 15.00 / 0$
Several lines of evidence suggest that prolonged sleep deprivation affects a wide network of brain structures. Neurons in several wake-promoting nuclei display continuous high-frequency activity throughout wakefulness. These include noradrenergic neurons in the locus ceruleus, serotoninergic neurons in the raphe nuclei, cholinergic neurons in both the pedunculopontine tegmentum and basal forebrain, glutamatergic neurons in the midbrain, histaminergic neurons in the posterior hypothalamus, and orexinergic neurons in the lateral hypothalamus (Brown et al., 2012). The long, highly arborized axons and electrophysiological activity patterns of these neurons are associated with high energy demands and consequently are highly vulnerable to sleep deprivation with respect to maintaining mitochondrial metabolic homeostasis (Tononi and Cirelli, 2014). For similar reasons, the cortex is also a likely candidate to experience negative effects of sleep deprivation (Tononi and Cirelli, 2014). Finally, sleep deprivation can lead to obesity (Schmid et al., 2014), and several studies involving obese patients and animal models of obesity have reported neural inflammation and injuries in the hypothalamus (Thaler et al., 2013), thereby providing additional evidence of a link between insufficient sleep and neurodegeneration.

In an attempt to gain more insights on this relationship, Zhang et al. (2014) studied the effect of extended wakefulness on the metabolic homeostasis of locus ce- ruleus neurons (LCns) in mice. The authors subjected mice to two distinct sleepdeprivation protocols. The first protocol (called Sh-wake) consisted of a $3 \mathrm{~h}$ period of sleep deprivation from ZT8 to ZT11 (in this nomenclature, ZT0 refers to the beginning of the inactive period for nocturnal mice and corresponds to the time at which the lights are turned on). The second protocol (called Ext-wake) consisted of prolonged sleep deprivation for $8 \mathrm{~h}$ (from ZT3 to ZT11) repeated for 3 consecutive days. Following the Sh-wake condition, several antioxidant proteins, including superoxide dismutase 2 and catalase, were upregulated in the LCns. Surprisingly, however, this homeostatic redox response was not activated in mice that were subjected to the Ext-wake protocol. This resulted in oxidative stress in the LCns, as shown by a significant increase in levels of the reactive oxygen species (ROS) superoxide $\left(\mathrm{O}_{2}^{-\cdot}\right)$ in the LCns (Zhang et al., 2014, their Fig. 1). Remarkably, the Ext-wake protocol led to the loss of $\sim 30 \%$ of LCns, and the remaining neurons had signs of ongoing neurodegenerative processes (Zhang et al., 2014, their Fig. 7). Zhang et al. (2014) reported similar signs of oxidative stress in the frontal cortex of mice subjected to the Ext-wake protocol (their Fig. 3). These findings suggest that, together with other possible factors, sleep deprivation could be a significant causative factor in the development of neurodegenerative diseases. Consistent with this conclusion, a recent study reported that fragmented sleep patterns 
increase the risk of Alzheimer's disease and can accelerate age-related cognitive decline and the development of neurofibrillary tangle pathology in individuals carrying the apolipoprotein E $\varepsilon 4$ allele (Lim et al., 2013).

SIRT3 is a member of a family of $\mathrm{NAD}^{+}$-dependent deacetylase enzymes localized in the mitochondrial inner membrane that are important regulators of cellular energy homeostasis (Nogueiras et al., 2012). The oxidative stress-related sensitivity of SIRT3 in other conditions such as aging (for review, see Nogueiras et al., 2012) prompted Zhang et al. (2014) to hypothesize that the adaptive metabolic homeostasis response in the LCns following sleep deprivation might be mediated through SIRT3-dependent pathways. Indeed, the divergence of the antioxidant response in LCns between mice subjected to the Sh-wake and mice subjected to the Ext-wake protocol were mirrored in the activity pattern of SirT3-dependent pathways in the LCns, which were upregulated in Sh-wake and downregulated in Extwake animals (Zhang et al., 2014, their Fig. 2). To further test the role of SIRT3 in the response of LCns, Zhang et al. (2014) exposed SirT3-knock-out (SirT3 ${ }^{-1-}$ ) mice to the Sh-wake and Ext-wake sleep deprivation protocols. The redox homeostatic response in LCns that was found in wild-type control-littermates was absent in Sh-wake-subjected SirT3 ${ }^{-/-}$mice (Zhang et al., 2014, their Fig. 5). Counterintuitively, Ext-wake-treated $\mathrm{SirT3}^{-1-}$ mice do not have an exacerbated neurodegeneration of LCns. However, compared with wild-type, $\mathrm{SirT3}^{-/-}$mice have already $\sim 30 \%$ fewer LCns under normal baseline conditions (Zhang et al., 2014, their Fig. 7).

These results provide compelling evidence that Sirt3-dependent pathways are both involved in and required for the maintenance of neuroprotective redox homeostasis responses following sleep deprivation. However, the mechanism that links sleep loss with impaired Sirt3 signaling and the downstream initiation of neurodegenerative processes remains unknown. Results from a recent groundbreaking study (Xie et al., 2013) may shed light on this question. Xie et al. (2013) observed vigilance state-dependent changes in the interstitial space within the brain. Compared with awake animals, a $60 \%$ increase in the interstitial space was recorded during sleep. Through this increase, the exchange rate between cerebrospinal and interstitial fluids increased during sleep, thereby enhancing the clearance of waste products that accumulate in

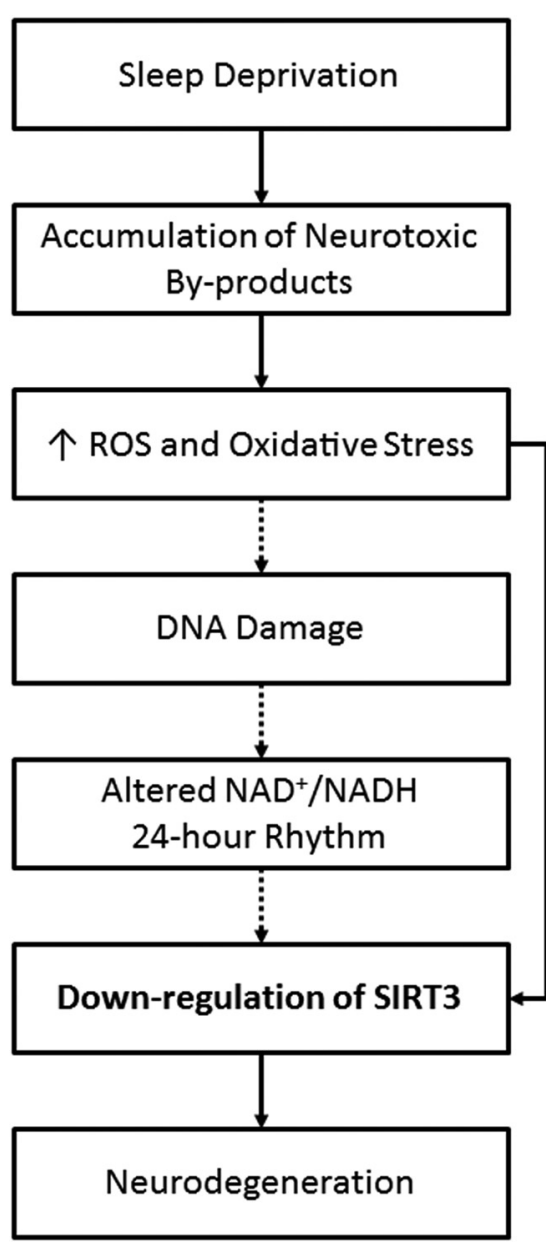

Figure 1. Impairment in the SIRT3 pathway following sleep deprivation leads to neurodegeneration. The accumulation of neurotoxic by-products in the brain during extended wakefulness (i.e., sleep deprivation) (Xie et al., 2013) leads to the downregulation of SIRT3-dependent pathways either directly through ROS (Weir et al., 2012) or indirectly (dashed vertical arrows) by impairing the circadian NAD ${ }^{+} / \mathrm{NADH}$ cycle (Peek et al., 2013). The ultimate consequence of this downregulation is neurodegeneration.

the brain during wakefulness (Xie et al., 2013). The accumulation of potentially neurotoxic by-products during extended sleep deprivation might impair the SIRT3 pathway either directly through ROS (Weir et al., 2012) or indirectly through impaired $\mathrm{NAD}^{+}$biosynthesis as a consequence of DNA damage (Fig. 1; Nogueiras et al., 2012). The latter mechanism might seem unlikely in light of the increase in cellular $\mathrm{NAD}^{+}$in Ext-wake-subjected mice reported by Zhang et al. (2014) in their Figure 4. However, given that the $\mathrm{NAD}^{+}$assays were performed at only one time point during a $24 \mathrm{~h}$ period, a change in the circadian pattern of $\mathrm{NAD}^{+}$biosynthesis - which is the main driver of SIRT3 activity (Peek et al., 2013) — cannot be excluded. Indeed, this scenario is possible, given the well documented negative- feedback effect of sleep deprivation on the central clock in the suprachiasmatic nucleus (Schmidt et al., 2009), which in turn drives the circadian $\mathrm{NAD}^{+}$cycle (Peek et al., 2013). Therefore, assessing the effect of sleep deprivation on the circadian pattern of $\mathrm{NAD}^{+}$bioavailability will be a key step in determining the relative contribution of impaired $\mathrm{NAD}^{+}$biosynthesis to SIRT3 dysfunction.

From a therapeutic perspective, the findings of Zhang et al. (2014) may serve as a catalyst in the development of drugs that directly modulate SIRT3-dependent pathways. Unlike SIRT1, for which a number of synthetic modulators have already yielded positive preclinical results (Nogueiras et al., 2012) and are currently being tested in patients with metabolic and neurodegenerative disorders $(\mathrm{NIH}$; www.clinicaltrials.gov), SIRT3 is still largely unexplored as a drug target (Nogueiras et al., 2012). Given that the activity of SIRT3 is modulated by $\mathrm{NAD}^{+}$levels, one promising avenue for counteracting the negative effects of sleep loss is $\mathrm{NAD}^{+}$ supplementation. However, the efficacy of this approach will depend on precisely how the circadian cycle of $\mathrm{NAD}^{+}$availability is affected by sleep deprivation.

Together, the data presented by Zhang et al. (2014) provide compelling evidence that SIRT3-dependent pathways are a putative molecular link between sleep loss and neurodegeneration. Hopefully, these seminal findings will inspire future research to help unravel the neuronal and molecular mechanisms that underlie the widespread and diverse family of neurological disorders that are linked to sleep deprivation.

\section{References}

Brown RE, Basheer R, McKenna JT, Strecker RE, McCarley RW (2012) Control of sleep and wakefulness. Physiol Rev 92:1087-1187. CrossRef Medline

Costandi M (2013) Neurodegeneration: amyloid awakenings. Nature 497:S19-S20. CrossRef Medline

Lim AS, Yu L, Kowgier M, Schneider JA, Buchman AS, Bennett DA (2013) Modification of the relationship of the apolipoprotein E $\varepsilon 4$ allele to the risk of Alzheimer disease and neurofibrillary tangle density by sleep. JAMA Neurol 70:1544-1551. CrossRef Medline

Nogueiras R, Habegger KM, Chaudhary N, Finan B, Banks AS, Dietrich MO, Horvath TL, Sinclair DA, Pfluger PT, Tschöp MH (2012) Sirtuin 1 and sirtuin 3: physiological modulators of metabolism. Physiol Rev 92:14791514. CrossRef Medline

Palma JA, Urrestarazu E, Iriarte J (2013) Sleep loss as risk factor for neurologic disorders: a review. Sleep Med 14:229-236. CrossRef Medline 
Peek CB, Affinati AH, Ramsey KM, Kuo HY, Yu W, Sena LA, Ilkayeva O, Marcheva B, Kobayashi Y, Omura C, Levine DC, Bacsik DJ, Gius D, Newgard CB, Goetzman E, Chandel NS, Denu JM, Mrksich M, Bass J (2013) Circadian clock NAD + cycle drives mitochondrial oxidative metabolism in mice. Science 342:1243417. CrossRef Medline

Perry GS, Patil SP, Presley-Cantrell LR (2013) Raising awareness of sleep as a healthy behavior. Prev Chronic Dis 10:E133. CrossRef Medline

Schmid SM, Hallschmid M, Schultes B (2014) The metabolic burden of sleep loss. Lancet Diabetes Endocrinol. Advance online publication. Retrieved March 25, 2014. doi:10.1016/ S2213-8587(14)70012-9. CrossRef Medline

Schmidt C, Collette F, Leclercq Y, Sterpenich V, Vandewalle G, Berthomier P, Berthomier C,
Phillips C, Tinguely G, Darsaud A, Gais S, Schabus M, Desseilles M, Dang-Vu TT, Salmon E, Balteau E, Degueldre C, Luxen A, Maquet P, Cajochen C, Peigneux P (2009) Homeostatic sleep pressure and responses to sustained attention in the suprachiasmatic area. Science 324: 516-519. CrossRef Medline

Thaler JP, Guyenet SJ, Dorfman MD, Wisse BE, Schwartz MW (2013) Hypothalamic inflammation: marker or mechanism of obesity pathogenesis? Diabetes 62:2629-2634. CrossRef Medline

Tononi G, Cirelli C (2014) Sleep and the price of plasticity: from synaptic and cellular homeostasis to memory consolidation and integration. Neuron 81:12-34. CrossRef Medline

Wade AG (2010) The societal costs of insomnia. Neuropsychiatr Dis Treat 7:1-18. CrossRef Medline
Weir HJ, Murray TK, Kehoe PG, Love S, Verdin EM, O'Neill MJ, Lane JD, Balthasar N (2012) CNS SIRT3 expression is altered by reactive oxygen species and in Alzheimer's disease. PLoS One 7:e48225. CrossRef Medline

Xie L, Kang H, Xu Q, Chen MJ, Liao Y, Thiyagarajan M, O'Donnell J, Christensen DJ, Nicholson C, Iliff JJ, Takano T, Deane R, Nedergaard M (2013) Sleep drives metabolite clearance from the adult brain. Science 342:373-377. CrossRef Medline

Zhang J, Zhu Y, Zhan G, Fenik P, Panossian L, Wang MM, Reid S, Lai D, Davis JG, Baur JA, Veasey S (2014) Extended wakefulness: compromised metabolics in and degeneration of locus ceruleus neurons. J Neurosci 34:4418-4431. CrossRef Medline 